\title{
Comparison of Videofluoroscopic Swallowing Study in Patients with Stroke-Associated Pneumonia
}

\author{
Jae Sam Seo, M.D., Kyo Hun Ku, M.D., Young Sook Park, M.D., Ph.D \\ Department of Physical Medicine and Rehabilitation, Samsung Changwon Hospital, Sungkyunkwan University School \\ of Medicine, Changwon, Korea
}

\begin{abstract}
Objective: To compare the findings of videofluoroscopic swallowing study (VFSS) in patients with stroke-associated pneumonia (SAP).

Methods: Between January 2015 and June 2019, 1245 patients diagnosed with pneumonia underwent VFSS examination. Data were collected through retrospective chart reviews. Depending on the diagnosis, 378 post-stroke patients identified were classified into two groups: 327 non-aspiration pneumonia (non-AP) and 51 aspiration pneumonia (AP). Aspiration pneumonia was diagnosed both clinically and radiologically. VFSS results included 11 items of functional dysphagia scoring (FDS) and 8 points of penetration-aspiration scale (PAS) that analyze the swallowing function by anatomical site.

Results: Based on the FDS score, the AP group showed significantly higher values in residue in pyriform sinuses ( $\mathrm{P}=$ $0.01)$, as compared to the non-AP group. There was a significant difference in the PAS score between both groups $(\mathrm{P}<0.001)$.

Conclusion: Based on the FDS results, AP in SAP is associated with residue in piriform sinuses. VFSS tests therefore have the potential to be clinically applied to predict the occurrence of aspiration pneumonia in stroke patients. (JKDS 2021;11:52-58)
\end{abstract}

Keywords: Stroke, Aspiration, Pneumonia

\section{INTRODUCTION}

Stroke-associated pneumonia (SAP) is a frequent and an important medical condition relation to significant mortality and financial burden after stroke $^{1}$. There are two pathophysiological mechanisms of SAP: aspiration theory vs stroke induced immunodepression theory ${ }^{2}$. Traditionally, SAP is thought to be secondary to aspiration related to dys- phagia and impaired consciousness. It has been suggested that abnormal dopamine transmission and low substance P level would be involved in impaired swallowing mechanism leading to aspiration of oral content during sleep ${ }^{3,4}$. Stroke induced immunodepression is thought to be the result of sympathetic system, parasympathetic system, and hypothalamicpituitary-adrenal axis activation ${ }^{5-7}$. The activation is prominent among patients with large stroke and have
Received: July 27 2020, Revised: July 28 2020,

Accepted: August 272020

Corresponding author: Young Sook Park, Department of Physical Medicine and Rehabilitation, Samsung Changwon Hospital, Sungkyunkwan University School of Medicine, 158 Paryong-ro, Hapseong-dong, Masanhoewon-gu, Changwon 51353, Korea

Tel: +82-55-233-6495, Fax: +82-55-233-5454

E-mail: jijibaeheiwon@hanmail.net
Copyrights (c) The Korean Dysphagia Society, 2021. 
predilection for stroke location such as the involvement of the insular cortex ${ }^{8,9}$.

Many studies reported about risk factors of SAP such as stroke severity measured by the NIHSS or the modified Rankin Scale, old age, mechanical ventilation, male sex, atrial fibrillation, dysarthria, comorbid medical condition, etc ${ }^{10,11}$. SAP is a complex disease and multiple factors are involved in the pathogenesis. Recently, many studies describe the clinical features and outcomes of patients with aspiration pneumonia (AP) compared to those of patients with non-aspiration pneumonia (non-AP) in $\mathrm{SAP}^{12}$. However, there was no study about comparing the videofluoroscopic swallow study (VFSS) finding between AP and non-AP in SAP patients. The VFSS has been the gold standard for evaluating patients with swallowing disorders for many years ${ }^{13}$. This method not only estimates the risks of aspiration and respiratory complications but also helps determine diet and compensatory strategies. Functional dysphagia scale (FDS) and penetration-aspiration scale (PAS) based on VFSS could be used to quantify functional dysphagia such as the oropharyngeal and esophageal phases of swallowing. Moon et al. reported that characteristic of VFSS findings in patients diagnosed with AP comparing with non-AP in neurologic deficit patients including central nervous system injury, neuromuscular disease, and neurodegenerative disease ${ }^{14}$. They reported that there were no significant differences in VFSS findings scored using the PAS and Pharyngeal Residue Grade (PRG) between groups. In stroke patients, however, it could be predicted that the results of the VFSS test would vary depending on the pathomechanism of SAP.

The aim of our study was to compare the VFSS findings between AP and non-AP in stroke patients.

\section{MATERIALS AND METHODS}

\section{Subjects}

This was a retrospective study that reviewed medical records and the results of VFSS who were referred for swallowing study to evaluate the cause of pneumonia between January 2015 and June 2019. Exclusion criteria of subjects were as follows: 1) diagnosis was not stroke, 2) the diagnosis of pneumonia was not clear, and 3) incomplete swallowing study. SAP patients were divided into two groups depending on the diagnosis: non-aspiration pneumonia (nonAP) and aspiration pneumonia (AP). Aspiration pneumonia group was classified based on records of receiving antibiotics treatment after diagnosis of aspiration pneumonia in consultation with internal medicine. Physicians diagnosed aspiration pneumonia based on clinical symptoms, images, and blood test results.

Flowchart of study subjects was showed in Fig. 1. This study was approved by the Samsung Changwon Hospital Institutional Review Board (No. 2020-SCMC070-13).

\section{VIdeofluoroscopic swallowing study}

VFSS test was done by fluoroscopic X-ray (sonial vision G4, shimadzu, Kyoto, Japan) according to protocol standardized by developing a sequence of liquid and solid foods, starting with those that are easiest for most patients to swallow, and progressing to more difficult consistencies.

\section{Functional dysphagia scale (FDS) measures}

The FDS was devised to quantify the results of the VFSS test by Han et al. ${ }^{13}$. Lip closure (intact 0 , inadequate 5, none 10), bolus formation (intact 0 , inadequate 3 , none 6 ), residue in oral cavity (none $0, \leq 10 \% 2,10 \%-50 \% 4, \geq 50 \% 6$ ), and oral transit time ( $\leq 1.5 \mathrm{~s} 0,>1.5 \mathrm{~s} 6$ ) constitute an oral phase. Triggering of pharyngeal swallow (normal 0 , delayed 10), laryngeal elevation and epiglottic closure (normal 0 , reduced 12 ), nasal penetration (none $0, \leq 10 \% 4$, $10 \%-50 \% 8, \geq 50 \% 12$ ), residue in valleculae (none 0 , $\leq 10 \% 4,10 \%-50 \% 8, \geq 50 \% 12$ ), residue in pyriform sinuses (none $0, \leq 10 \% 4,10 \%-50 \% \quad 8, \geq 50 \% 12$ ), coating of pharyngeal wall after swallow (no 0 , yes $10)$, and pharyngeal transit time ( $\leq 1.0$ s $0,1.0>$ s 4$)$ constitute pharyngeal phase. The total score is 100 and the higher the score, the more severe the dys- 


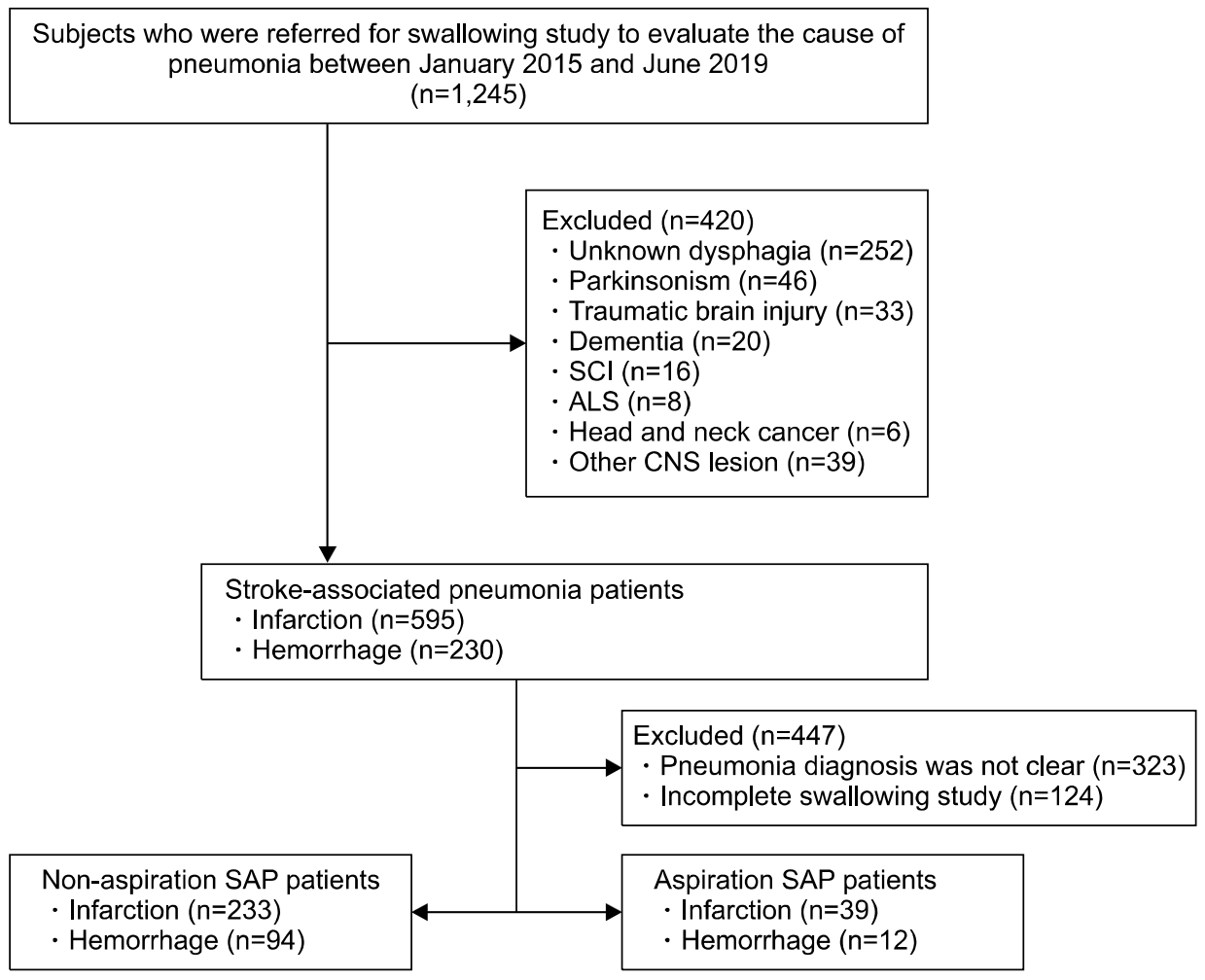

Fig. 1. Flowchart of subjects.

Table 1. Demographic characteristics of subjects with pneumonia.

\begin{tabular}{|c|c|c|c|}
\hline & Non-aspiration $(n=327)$ & Aspiration $(\mathrm{n}=51)$ & P-value \\
\hline Age (years) & $72.63 \pm 12.11$ & $74.76 \pm 10.84$ & 0.235 \\
\hline Sex & & & 0.001 \\
\hline Female & $168(51.38)$ & $39(76.47)$ & \\
\hline Male & $159(48.62)$ & $12(23.53)$ & \\
\hline Disease & & & 0.440 \\
\hline Cerebral infarction & $233(71.25)$ & $39(76.47)$ & \\
\hline Cerebral hemorrhage & $94(28.75)$ & $12(23.53)$ & \\
\hline NIHSS (n=127) & $7.75 \pm 6.19$ & $10.00 \pm 5.42$ & 0.158 \\
\hline MMSE $(n=101)$ & $14.07 \pm 9.63$ & $16.13 \pm 9.07$ & 0.442 \\
\hline VFSS score total & $32.95 \pm 12.69$ & $38.55 \pm 12.00$ & 0.003 \\
\hline PAS score & $5.03 \pm 2.49$ & $6.75 \pm 2.00$ & $<0.001$ \\
\hline
\end{tabular}

Continuous variables are presented as mean \pm standard deviation (SD), and categorical variables are presented as number and percent. Independent samples t-test or $\chi^{2}$-test as appropriate. Bold number means $\mathrm{P}<0.05$ and significant difference.

phagia.

\section{Penetration-aspiration scale (PAS) measures}

The PAS is an 8-point, equal-appearing interval scale to describe penetration and aspiration events ${ }^{15}$. Scores are determined primarily by the depth to which material passes in the airway and by whether or not material entering the airway is expelled.

Higher score means a poorer swallowing function.

\section{Statistical analysis}

Statistical analysis was performed using STATA 14.0 (Stata Corporation, College Station, TX, USA), and average and standard deviation of the data were obtained using the descriptive statistics. We compared differences between the two groups using independent samples t-test or Fisher's exact test or Pearson's chi-square test as appropriate. The P-value 
of $<0.05$ was considered to be statistically significant.

\section{RESULTS}

Data from a total of 1,245 subjects who were referred for swallowing study to evaluate swallowing problems or dysphagia that might cause pneumonia

Table 2. Comparison for functional dysphagia scale using VFSS.

\begin{tabular}{|c|c|c|c|}
\hline & Non-aspiration $(\mathrm{n}=327)$ & Aspiration $(n=51)$ & P-value \\
\hline Lip closure & & & $>0.999$ \\
\hline 0 & $318(97.25)$ & $50(98.04)$ & \\
\hline 5 & $9(2.75)$ & $1(1.96)$ & \\
\hline 10 & $0(0.00)$ & $0(0.00)$ & \\
\hline Bolus formation & & & 0.229 \\
\hline 0 & $52(15.90)$ & $5(9.80)$ & \\
\hline 3 & $273(83.49)$ & $45(88.24)$ & \\
\hline 6 & $2(0.61)$ & $1(1.96)$ & \\
\hline Residue in oral cavity & & & 0.372 \\
\hline 0 & $72(22.02)$ & $8(15.69)$ & \\
\hline 2 & $219(66.97)$ & $38(74.51)$ & \\
\hline 4 & $30(9.17)$ & $3(5.88)$ & \\
\hline 6 & $6(1.83)$ & $2(3.92)$ & \\
\hline Oral transit time & & & 0.524 \\
\hline 0 & $224(68.50)$ & $33(64.70)$ & \\
\hline 6 & $103(31.49)$ & $18(35.29)$ & \\
\hline Triggering of pharyngeal swallow & & & 0.225 \\
\hline 0 & $148(45.26)$ & $18(35.29)$ & \\
\hline 10 & $179(54.74)$ & $33(64.70)$ & \\
\hline Laryngeal elevation and epiglottic closure & & & $>0.999$ \\
\hline 0 & $6(1.83)$ & $0(0.00)$ & \\
\hline 12 & $321(98.17)$ & $51(100.00)$ & \\
\hline Nasal penetration & & & 0.191 \\
\hline 0 & $314(96.02)$ & $46(90.20)$ & \\
\hline 4 & $12(3.67)$ & $5(9.80)$ & \\
\hline 8 & $1(0.31)$ & $0(0.00)$ & \\
\hline 12 & & & \\
\hline Residue in valleculae & & & 0.060 \\
\hline 0 & $99(30.28)$ & $11(21.57)$ & \\
\hline 4 & $215(65.75)$ & $35(68.63)$ & \\
\hline 8 & $13(3.98)$ & $4(7.84)$ & \\
\hline 12 & $0(0.00)$ & $1(1.96)$ & \\
\hline Residue in pyriform sinuses & & & 0.014 \\
\hline 0 & $158(48.32)$ & $16(31.37)$ & \\
\hline 4 & $148(45.26)$ & $26(50.98)$ & \\
\hline 8 & $19(5.81)$ & $8(15.69)$ & \\
\hline 12 & $2(0.61)$ & $1(1.96)$ & \\
\hline Coating of pharyngeal wall after swallow & & & 0.059 \\
\hline 0 & $273(83.49)$ & $37(72.55)$ & \\
\hline 10 & $54(16.51)$ & $14(27.45)$ & \\
\hline Pharyngeal transit time & & & 0.119 \\
\hline 0 & $147(44.95)$ & $17(33.33)$ & \\
\hline 4 & $180(55.05)$ & $34(66.67)$ & \\
\hline Total & $32.95 \pm 12.69$ & $38.55 \pm 12.00$ & 0.003 \\
\hline
\end{tabular}

Categorical variables are presented as number and percent. Fisher's exact test or Pearson's chi-square test as appropriate. Bold number means $\mathrm{P}<0.05$ and significant difference. 


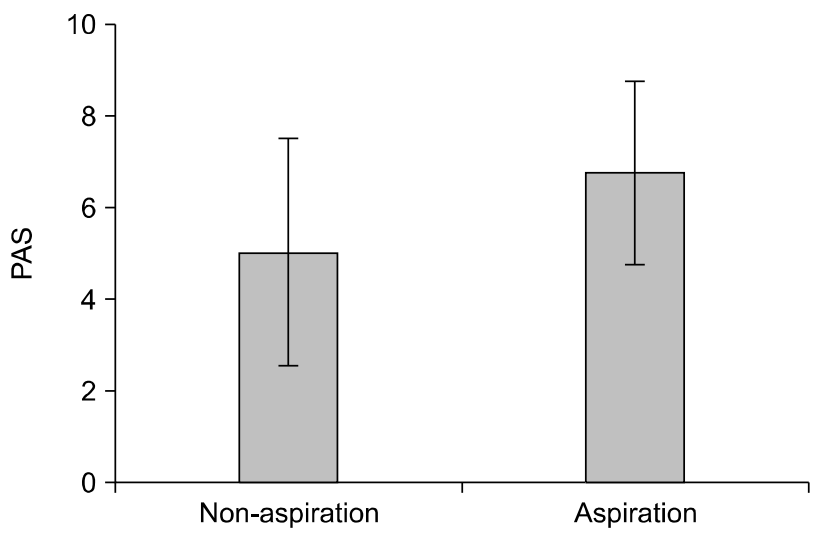

Fig. 2. Comparison for penetration-aspiration scale using VFSS.

between January 2015 and June 2019 were considered for analysis. Initially, 420 subjects whose diagnosis was not stroke were excluded. And then, 447 subjects were excluded because of unclear pneumonia diagnosis and incomplete swallowing study. Finally, 378 subjects included in analysis comparing non-AP and AP in stroke patients.(Fig. 1) The gender and dysphagia score were statistically significant between non-AP and AP groups in demographic characteristics.(Table 1) Based on the FDS score, the AP group showed significantly higher values in residue in pyriform sinuses $(\mathrm{P}=0.014)$ compared with non-AP group. (Table 2) The PAS score of non-AP group and AP group was $5.03 \pm 2.49$ and $6.75 \pm 2.00$, respectively. There was a significant difference between two groups $(\mathrm{P}<0.001)$ as showed in Fig. 2.

\section{DISCUSSION}

In this study, we compared FDS score and PAS score of AP patients with that of non-AP patients based on VFSS in stroke patients. Residue in pyriform sinuses based on FDS was higher score in AP group compared with non-AP group. In previous studies, stroke survivors with penetration and aspiration tended to have more vallecular and pyriform sinus residue than other group and residue of food in the pharynx raised the risk of aspiration ${ }^{16,17}$. This might be because the stroke impaired motor and sensory control of the muscle that involved in swallowing.
Mann et al. reported that more than $30 \%$ of poststroke survivors showed weak sensorimotor control of tongue and submental muscles during the oral stage of swallowing ${ }^{18}$. Stokely et al. reported that increased post-swallowing residue in the valleculae and pyriform sinuses had correlation with increased pharyngeal area at maximal contraction ${ }^{19}$. The results of our study were consistent with those in previous study, however, compared to previous studies, the significance of this study was that these changes showed a significant difference in patients with AP compared to those with non-AP among SAP. Stroke-associated infection mainly includes SAP and urinary tract infections (UTI) and overall infection rate is $30 \%$. UTI and SAP show a similar prevalence, whereas SAP is significantly associated with death ${ }^{20}$. Many factors influence SAP, however, based on accumulating evidence, changes in immunity triggered by an acute cerebral injury is the main cause of $\mathrm{SAP}^{21}$. Xabier et al. reported that infarct volume has an association with the occurrence of stroke-associated infections and specific brain locations such as the superior and lateral temporal lobe and the orbitofrontal cortex are also associated with increased infectious risk, especially pneumonia ${ }^{22}$. In our study, we could not identify whether or not pneumonia developed according to anatomical location because stroke was classified simply as cerebral hemorrhage and infarction. PAS score was significantly higher in AP group than nonAP group in SAP as observed in other studies of dysphagia. However, PAS score had limitation to providing anatomical information regarding aspiration. According to the results of the previous study of clinical differences between patients with AP and non-AP, AP were frequent residence in a nursing home, long-term facility, more severe form of pneumonia, and poorer outcome ${ }^{23}$. If the VFSS test could identify and prevent the possibility of aspiration pneumonia, many prognosis factors such as length of hospitalization and mortality for stroke patients will improve. Thus, early performance of the VFSS after stroke could facilitate the determination of appropriate feeding methods and prevent aspiration and 
improve of quality of life for stroke patients.

This study had some limitations. First, ischemic and hemorrhagic stroke patients were analyzed together. We could not classify the possibility of aspiration by type of stroke. Second, the results of our study were consistent with the previously reported findings of studies that used similar designs. However, we only included stroke patients to compare the results of VFSS between AP and non-AP. Third, patient comorbidities were not analyzed. That could affect the possibility of aspiration and also the changes in VFSS results.

\section{CONCLUSION}

The pathophysiology of SAP is likely explained by aspiration combined with stroke-induced immunodepression through complex humeral and neural pathways that include the hypothalamic-pituitaryadrenal axis, parasympathetic and sympathetic systems. However, AP in SAP was associated with residue in piriform sinuses based on FDS in the current study. The authors speculated that it would be clinically useful to predict the occurrence of aspiration pneumonia in stroke patients with VFSS tests.

\section{CONFLICT OF INTEREST}

No potential conflict of interest relevant to this article was reported.

\section{ACKNOWLEDGEMENTS}

This study was approved by the Samsung Changwon Hospital Institutional Review Board (No. 2020SCMC-070-13).

\section{REFERENCES}

1. Koennecke HC, Belz W, Berfelde D, Endres M, Fitzek S, Hamilton F, et al. Factors influencing in-hospital mortality and morbidity in patients treated on a stroke unit. Neurology. 2011;77:965-72.

2. Hannawi Y, Hannawi B, Rao CP, Suarez JI, Bershad EM.
Stroke-associated pneumonia: major advances and obstacles. Cerebrovasc Dis. 2013;35:430-43.

3. Teramoto S. Novel preventive and therapuetic strategy for post-stroke pneumonia. Expert Rev Neurother. 2009; 9:1187-200

4. Nakagawa T, Ohrui T, Sekizawa K, Sasaki H. Sputum substance $\mathrm{P}$ in aspiration pneumonia. Lancet. 1995;345: 1447.

5. Chamorro A, Meisel A, Planas AM, Urra X, van de Beek D, Veltkamp R. The immunology of acute stroke. Nat Rev Neurol. 2012;8:401-10.

6. Dirnagl U, Klehmet J, Braun JS, Harms H, Meisel C, Ziemssen $\mathrm{T}$, et al. Stroke-induced immunodepression: experimental evidence and clinical relevance. Stroke. 2007:38:770-3.

7. Meisel C, Schwab JM, Prass K, Meisel A, Dirnagl U. Central nervous system injury-induced immune deficiency syndrome. Nat Rev Neurosci. 2005;6:775-86.

8. Liesz A, Hagmann S, Zschoche C, Adamek J, Zhou W, Sun $\mathrm{L}$, et al. The spectrum of systemic immune alterations after murine focal ischemia: immunodepression versus immunomodulation. Stroke. 2009;40:2849-58.

9. Walter U, Kolbaske S, Patejdl R, Steinhagen V, AbuMugheisib M, Grossmann A, et al. Insular stroke is associated with acute sympathetic hyperactivation and immunodepression. Eur J Neurol. 2013;20:153-9.

10. Walter U, Knoblich R, Steinhagen V, Donat M, Benecke $\mathrm{R}$, Kloth A. Predictors of pneumonia in acute stroke patients admitted to a neurological intensive care unit. $\mathrm{J}$ Neurol. 2007;254:1323-9.

11. Weimar C, Roth MP, Zillessen G, Glahn J, Wimmer ML, Busse $\mathrm{O}$, et al. Complications following acute ischemic stroke. Eur Neurol. 2002;48:133-40.

12. Yu KJ, Moon H, Park D. Different clinical predictors of aspiration pneumonia in dysphagic stroke patients related to stroke lesion: A STROBE-complaint retrospective study. Medicine (Baltimore). 2018;97:e13968.

13. Han TR, Paik NJ, Park JW. Quantifying swallowing function after stroke: A functional dysphagia scale based on videofluoroscopic studies. Arch Phys Med Rehabil. 2001; 82:677-82.

14. Moon MH, Min JH, Shin YI, Ko SH. Frequency and characteristics of videofluoroscopic swallow study in patients with aspiration pneumonia. J Korean Dysphagia Soc. 2018; 8:48-55.

15. Rosenbek JC, Robbins JA, Roecker EB, Coyle JL, Wood JL. A penetration-aspiration scale. Dysphagia. 1996;11: 93-8.

16. Han H, Shin G, Jun A, Park T, Ko D, Choi E, et al. The Relation Between the Presence of Aspiration or Penetration and the Clinical Indicators of Dysphagia in Poststroke Survivors. Ann Rehabil Med. 2016;40:88-94.

17. Daniels SK, Schroeder MF, McClain M, Corey DM, Rosenbek JC, Foundas AL. Dysphagia in stroke: Development of a standard method to examine swallowing recovery. J Rehabil Res Dev. 2006;43:347-56. 
18. Mann G, Hankey GJ, Cameron D. Swallowing function after stroke: prognosis and prognostic factors at 6 months. Stroke. 1999;30:744-8.

19. Stokely SL, Peladeau-Pigeon M, Leigh C, Molfenter SM, Steele CM. The Relationship Between Pharyngeal Constriction and Post-swallow Residue. Dysphagia. 2015;30: 349-56.

20. Westendorp WF, Nederkoorn PJ, Vermeij JD, Dijkgraaf MG, van de Beek D. Post-stroke infection: a systematic review and meta-analysis. BMC Neurol. 2011;11:110.

21. Chamorro A, Urra X, Planas AM. Infection after acute ischemic stroke: a manifestation of brain-induced immunodepression. Stroke. 2007;38:1097-103.

22. Ryu WS, Woo SH, Schellingerhout D, Jang MU, Park KJ, Hong KS, et al. Stroke outcomes are worse with larger leukoaraiosis volumes. Brain. 2017;140:158-70.

23. Hayashi M, Iwasaki T, Yamazaki Y, Takayasu H, Tateno $\mathrm{H}$, Tazawa S, et al. Clinical features and outcomes of aspiration pneumonia compared with non-aspiration pneumonia: a retrospective cohort study. J Infect Chemother. 2014;20:436-42. 